\title{
Risk factors of transient neurological deficits and perioperative stroke after Revascularization in patients with moyamoya disease
}

xincheng zhang

Tongji Hospital

Yiping Yang

Tongji Hospital

Chao Gan

Tongji Hospital

Xuejun He

Tongji Hospital

Yanchao Liu

Tongji Hospital

Yimin Huang

Tongji Hospital

Xiaopeng $\mathrm{Ma}$

Tongji Hospital

Kai Shu

Tongji Hospital

Ting Lei

Tongji Hospital

Sheng Wang

Tongji Hospital

huaqiu zhang ( $\nabla$ zhanghq@tjh.tjmu.edu.cn )

Tongji Hospital

\section{Research Article}

Keywords: Moyamoya disease, Revascularization, Complications, Transient neurological deficits

Posted Date: February 16th, 2022

DOI: https://doi.org/10.21203/rs.3.rs-1313632/v1 
License: (c) (i) This work is licensed under a Creative Commons Attribution 4.0 International License. Read Full License 


\section{Abstract}

OBJECTIVE: To analyze the risk factors of transient neurological deficits (TND) and perioperative stroke in patients with MMD after extracranial-intracranial revascularization.

METHODS: A retrospective analysis of the clinical data of 183 patients with MMD undergoing 203 procedures EC-IC bypass operation from January 2018 to August 2020. According to whether TND and stroke occurred within 14 days after operation, univariate analysis and multivariate logistic regression were used.

RESULTS: TND occurred in 26 cases (12.8\%) of revascularization. The results of the univariate analysis showed that history of diabetes, multiple episodes of preoperative symptoms, lesions involving the posterior circulation, and high postoperative blood pressure are the risk factors of TND. Further multivariate logistic regression analysis showed that multiple episodes of preoperative symptoms $(P=0.016)$ and lesions involving the posterior circulation $(P=0.014)$ are the independent risk factors for TND. Perioperative stroke occurred in 12 cases (5.9\%). The results of the univariate analysis showed that older age, history of hypertension, preoperative cerebral infarction as the main symptom, lesions involving the posterior circulation, and High perioperative blood pressure are the risk factors of the perioperative stroke. The results of multivariate logistic regression analysis showed that preoperative cerebral infarction as the main symptom $(P=0.015)$ is an independent risk factor for perioperative stroke. Whether perioperative complications occurred was not related to the improvement of follow-up mRS score and long-term cerebral rehemorrhage.

CONCLUSIONS: Clinically, patients with MMD have multiple episodes of preoperative symptoms, lesions involving the posterior circulation, and preoperative cerebral infarction should be attached when undergoing revascularization.

\section{Introduction}

Moyamoya disease is a chronic progressive cerebral vascular occlusive disease of unknown causes. ${ }^{1}$ In recent years, with the improvement of diagnosis and treatment, especially in the high incidence of East Asian countries, the detection rate is increasing. ${ }^{2,3}$ Due to the amazing protective effect, EC-IC bypass become the first-line treatment for the patients with MMD. ${ }^{4}$ Surgery methods mainly includes direct, indirect, and combined revascularization. The combined revascularization can not only increase intracranial blood flow immediately but also form effective collateral circulation in the long term, which has been widely preformed all over the world. ${ }^{5,6}$ However, as a kind of preventive surgery, the complications such as new current ischemic and hemorrhagic stroke, subdural effusion, poor scalp healing and transient neurological deficits (TND) are still the major problem for neurosurgeons ${ }^{7,8}$. In this study, we studied the two most common complications: TND and perioperative stroke to analyze the underlying mechanism and provide a reference for clinical work in the future. 


\section{Methods}

\section{Patient Selection:}

A total of 183 patients and 203 cases of MMD revascularization were included. Inclusion criteria: $\triangle$ Patients with MMD admitted to our hospital from January 2018 to August 2020. खPatients with MMD who meet the diagnostic criteria released by the Research Committee on the Pathology and Treatment of Spontaneous Occlusion of the Circle of Willis from the Ministry of Health and Welfare of

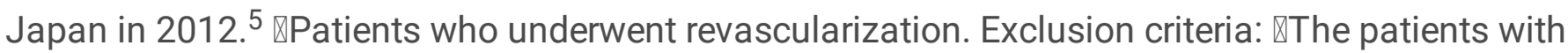
autoimmune diseases such as systemic lupus erythematosus, antiphospholipid antibody syndrome, Sjogren's syndrome, hyperthyroidism, tuberous sclerosis, Marfan syndrome, and so on. खThe patients were not diagnosed by DSA or MRA. $\triangle$ The patients were followed up for less than 3 months after discharge include death.

\section{Clinical data:}

The data of 21 clinical indicators were collected, including gender, age, history of hypertension, diabetes and aneurysm, the main clinical symptom of patients, Preoperative attack times, Interval between the last attack and operation, stage classification of MMD, lesions involving the posterior circulation, admission mRS score, operation method, operation side, times of bypass, duration of surgery, intraoperative blood loss, intraoperative $\mathrm{PCO}_{2}$, perioperative blood pressure (preoperative, intraoperative and postoperative), $24 \mathrm{~h}$ cranial drainage volume after operation, perioperative complications after operation, follow-up mRS score and long-term cerebral rehemorrhage (Tables 1 and 2 ).

\section{Definition of complications:}

Transient neurological deficits (TND): New symptoms of local neurological dysfunction during the perioperative period after operation, including aphasia, limb weakness or sensory abnormality, epilepsy, disturbance of consciousness, etc., There was no new responsible focus of stroke in imaging examination, and the symptoms recovered within 14 days or 1 month at the latest.

Perioperative stroke: New ischemic and hemorrhagic stroke events within 14 days after operation musthave new responsible lesions defined on imaging examination. There is no intersection between perioperative stroke and TND. Patients with TND first and then stroke was recorded as the perioperative stroke group because it was impossible to distinguish TND from the precursor of stroke.

Long-term cerebral hemorrhage: The new hemorrhagic stroke incident one month after surgery, which must have imaging (CT) evidence and exclude cerebral hemorrhage caused by trauma, ruptured aneurysm, and other causes that are not related to MMD.

\section{Definition of influencing factors:}


Main clinical symptom of patients: Refers to the most obvious symptom caused by MMD. Patients with both a preoperative history of cerebral infarction and cerebral hemorrhage were included in the cerebral hemorrhage group because cerebral hemorrhage is often more serious.

The interval between the last attack and operation: The patients in the acute operation group underwent operation within 1 month after the onset of cerebral infarction or within 3 months after the onset of a cerebral hemorrhage. The stable operation group was operated within 1 to 6 months after the onset of cerebral infarction, or within 3 to 6 months after the symptoms of a cerebral hemorrhage. The patients who underwent operation more than 6 months after the onset of stroke symptoms were regarded as the long-term operation group.

MMD stage: It refers to the staging on the side of the operation. Suzuki stage 1-2 or MRA score 0-1 is in the early stage, Suzuki stage 3-4 or MRA score 2-7 is in the middle stage, and Suzuki stage 5-6 or MRA score $8-10$ is in the late stage. ${ }^{5}$

Preoperative blood pressure: Patients with a history of hypertension had no blood pressure intervention and preoperative systolic blood pressure $\geq 140 \mathrm{mmHg}$ or diastolic blood pressure $\geq 90 \mathrm{mmHg}$ are recorded as preoperative high blood pressure.

Intraoperative blood pressure: Intraoperative high blood pressure was recorded when arterial systolic blood pressure $\geq 150 \mathrm{mmHg}$ or arterial diastolic blood pressure $\geq 100 \mathrm{mmHg}$.

Postoperative blood pressure: The systolic blood pressure $\geq 140 \mathrm{mmHg}$ or diastolic blood pressure $\geq 90 \mathrm{mmHg}$ measured randomly three times during the perioperative period after operation was regarded as postoperative high blood pressure.

$\mathrm{PCO}_{2}$ during operation: $\mathrm{PCO}_{2}<35 \mathrm{mmHg}$ measured at one-third of the time or twice randomly recorded during the operation as low $\mathrm{PCO}_{2}$ during the operation. $\mathrm{PCO}_{2}>45 \mathrm{mmHg}$ measured at one-third of the time or twice randomly recorded during the operation as high $\mathrm{PCO}_{2}$ during the operation.

\section{Operation:}

The surgical methods include combined revascularization and indirect revascularization. Indirect revascularization mainly refers to encepho-duro-myo-synangiosis (EDMS), Combined revascularization refers to the simultaneous implementation of extracranial-intracranial bypass surgery + EDMS.

\section{Follow-up method:}

Three months after surgery, the patient was followed up. All-inclusive patients were followed up for $\geq 3$ months (MD = 15 months, IQR = 9 months). The follow-up content includes Modified Rankin Scale (mRS) score and whether there is a long-term cerebral hemorrhage incident. 


\section{Statistical method:}

Statistical analysis of data using IBM SPSS Statistics version 25.0 (IBM Software Group, Chicago, IL, USA). The measurement data uses the $K$-S test for normal testing, and the measurement data that do not meet the normal distribution uses the $P-P$ grammar to determine whether they approximately obey the normal distribution or not. The measurement data which conformed to or approximately conformed to the normal distribution were expressed by mean \pm standard deviation ( ), and the differences between groups were compared by two independent samples $t$-test. The measurement data that do not conform to normal or approximate normal distribution are expressed by median and Inter Quartile Range (Md, IQR), and the differences between groups are compared by rank-sum test. The counting data were expressed by the number of cases and the rate. The chi-square test or Fisher's exact test was used to compare the differences between groups, and the Bonferroni correction was used for pairwise comparison within the group. Rank sum test was used for ordinal variable data. Multivariate analysis using binary logistic regression analysis. The $p$ values $<0.05$ were considered as statistically significant.

\section{Results}

\section{Incidence of complications:}

TND occurred in 26 cases (12.8\%) after cerebrovascular revascularization, of which aphasia (26.9\%) was the most common symptom. The median time of occurrence of TND was 3 days, and the median duration of TND was 6 days. There were 12 cases of perioperative stroke (5.9\%) and 8 cases of long-term cerebral hemorrhage (3.9\%) (Tables 3).

\section{Statistical Outcomes:}

Analysis of factors influencing the occurrence of perioperative complications: Univariate analysis showed that history of diabetes, multiple episodes of preoperative symptoms, lesions involving the posterior circulation, and high postoperative blood pressure were the risk factors of postoperative TND $(P<0.05)$. Meanwhile, older age, history of hypertension, the main clinical symptom of patients, lesions involving the posterior circulation, and High perioperative blood pressure are the risk factors of the perioperative stroke (Pख0.05). Further multivariate logistic regression analysis showed that multiple episodes of preoperative symptoms ( $\mathrm{OR}=3.134, \mathrm{Cl}: 1.236 \sim 7.943, \mathrm{P}=0.016)$ and lesions involving the posterior circulation (OR=0.327, $\mathrm{Cl}: 0.133 \sim 0.8, \mathrm{P}=0.014$ ) are an independent risk factor for TND. Meanwhile, the main clinical symptom of patients was an independent risk factor for perioperative stroke (OR=0.209, Cl: 0.059 0.738, $\mathrm{P}=0.015)$, and the pairwise comparison within the main clinical symptom group showed that the incidence of perioperative stroke in the preoperative cerebral infarction group was higher than that in the preoperative cerebral hemorrhage group $(P<0.0125)$ (Tables 4 and 5$)$.

\section{Follow-up results:}


There was no statistical difference in $\mathrm{mRS}$ between the perioperative complication group and the noncomplication group $(P=0.094)$. None of the 8 patients with long-term cerebral hemorrhage had perioperative complications, so perioperative complications had nothing to do with long-term cerebral hemorrhage $(P=0.37)$ (Tables 6).

\section{Discussion}

MMD is a rare cerebrovascular disease characterized by chronic progressive stenosis or occlusion of bilateral internal carotid arteries and secondary to the formation of an abnormal vascular network in the skull base. ${ }^{1}$ Its incidence is relatively high in East Asia, and the age distribution of patients conforms to the bimodal distribution, that is, children and young adults have a higher incidence, while females have a higher incidence than men. ${ }^{9}, 10$ However, the clinical data of our center do not show obvious age bimodal distribution characteristics and gender differences, which is consistent with the report of some Chinese Studies, ${ }^{11,12}$ which may be affected by the sample size and the misdiagnoses of Moyamoya in children. Compared with previous studies, the main clinical symptom of patients in this center was significantly higher in the cerebral hemorrhage group ( $45.3 \%)$, which may be related to the fact that the patients who had both hemorrhagic stroke symptoms and other symptoms were classified as the cerebral hemorrhage group. As the first-line therapy of MMD, cerebral revascularization can effectively protect patients, ${ }^{4}$ but its postoperative complications are more frequent and complex than other diseases undergoing EC-IC bypass surgery. ${ }^{7}$ Neurologic deterioration is a common complication of cerebral revascularization for the perioperative period in MMD, which can be divided into TND and stroke according to its reversibility and imaging findings.

\section{The mechanism of TND:}

At present, the reports on the mechanism and influencing factors of TND are still not comprehensive. It was previously reported that the incidence of TND after cerebral revascularization was about $9.4 \%-38.2 \%^{13-17}$, compared with $12.8 \%$ in this cohort, which is basically consistent with the reported studies. In the past, cerebral revascularization was considered to be a low-flow bypass, which was not easy to cause hyperperfusion changes. Early, Iwama et al. ${ }^{18}$ reported symptoms similar to TND but classified them as a kind of reversible ischemic neurologic deficits (RIND). They suggested that the mechanism of this symptom was caused by intracranial ischemia and was a precursor of cerebral infarction, but there was no imaging responsible focus. In 1998, Uno et al. ${ }^{19}$ reported for the first time the phenomenon of TND with hyperperfusion on SPECT in patients with MMD after EC-IC bypass, considering that the symptoms of TND in patients with MMD may be related to hyperperfusion. The subsequent scholars found hyperperfusion and local cerebral edema through SPECT and MRI and their time relationship with TND, confirmed that EC-IC bypass has the phenomenon of hyperperfusion, which may lead to hyperperfusion syndrome rather than ischemic injury, and this kind of symptom is called symptomatic hyperperfusion. ${ }^{14-16}$ Later, Hayashi et al. ${ }^{20}$ observed the decrease of regional cerebral perfusion far away from the anastomotic stoma on the SPECT of 15 children with TND, suggesting that 
watershed shift may also be the mechanism leading to TND. Yu et al. ${ }^{21}$ also reported a neurological disorder associated with watershed shift after EC-IC bypass in an adult female patient with MMD, but recently Tashiro et al. ${ }^{22}$ studied 7 cases of the watershed shift in 64 cases of MMD bypass surgery, but none of them was accompanied by deterioration of neurological function. The study of Mukerji et al. ${ }^{23}$ also proposed that the impairment of the self-regulating function of cerebral blood flow and local hypoperfusion caused by competitive blood flow may also be the cause of TND. The above studies have shown that changes in hemodynamics after EC-IC bypass are a mechanism that occurs in TND, but for indirect bypass, the 2 weeks after surgery can form a significant lateral recirculation, causing hemodynamics to change. However, in our study, TND occurred in 2 of the 7 patients with indirect bypass on the 2 nd and 6 th day after operation respectively. The explanation given by Phi et al. ${ }^{24}$ is that TND after indirect bypass may also be caused by the transient cortical depression triggered by mechanical stimulation rather than hyperperfusion. Therefore, can symptomatic hyperperfusion or cerebral hyperperfusion syndrome (CHS) be used instead of TND? The answer is no. TND is observed from symptomatology, while CHS is observed from imaging. The occurrence of TND does not necessarily have regional cerebral hyperperfusion, and it is observed that the presence of CHS does not automatically show clinical symptoms. The intersection of TND and CHS is symptomatic hyperperfusion, and CHS is more common than symptomatic hyperperfusion. ${ }^{25}$ Other mechanisms that may lead to TND include temporary occlusion of the recipient artery or the sacrifice of its small branches, the formation of microthrombus, and so on.

\section{The mechanism of perioperative cerebral infarction:}

It was previously reported that the incidence of perioperative cerebral infarction events was 3\% 10.3\% 26 30 , and our incidence was $4.9 \%$. It is now believed that the mechanism of perioperative ischemic stroke is related to watershed shift, perioperative management, and even the space-occupying effect of the temporal muscle. ${ }^{31}$ In the location of the stroke, surgical stroke is more common, contralateral or bilateral stroke is relatively rare. ${ }^{32}$ In our study, 10 patients with perioperative stroke were surgical stroke and 2 patients were bilateral stroke. We believe that the main cause of contralateral or even bilateral stroke is the blow of operation and anesthesia, which is less related to the disturbance of local perfusion after operation.

\section{The mechanism of perioperative cerebral hemorrhage:}

In the past, the incidence of perioperative cerebral hemorrhage reported was $0.13 \%-3.6 \%{ }^{28,33-35}$, which was similar to our result $(0.98 \%)$. In addition, our study excluded the cases of immediate anastomotic bleeding caused by inadequate surgical techniques, because these complications were caused by surgical procedures and had nothing to do with the risk factors we studied. The occurrence of hemorrhagic stroke is mainly related to hyperperfusion syndrome. ${ }^{35}$ In addition, severe transfer of watershed shift can also lead to hemorrhagic stroke. Ischemia-reperfusion injury after EC-IC bypass will increase the risk of bleeding. ${ }^{36}$ During the reperfusion of ischemic brain tissue, a variety of substances that can increase 
vascular permeability, including reactive oxygen species, vascular endothelial growth factor, and protease, may be released, which may lead to the increase of vascular permeability, leakage of plasma and red blood cells, and even vascular rupture and bleeding. ${ }^{37}$

In this study, we explored the effects of 19 factors on perioperative complications after revascularization of MMD and the relationship between perioperative complications and long-term prognosis. These factors were divided into four types: baseline data of patients, information of MMD, operation and anesthesia management, and perioperative management data.

\section{Baseline data of patients:}

Age: Our study found that older patients had a higher incidence of postoperative stroke events $(P<0.05)$, which may be due to the loss of self-regulating function of cerebral blood flow, or they are more sensitive to cerebral ischemia. ${ }^{12}$

History of hypertension: The history of hypertension is also related to postoperative stroke events. The accelerated hardening of intracranial vessels and the decrease of cerebrovascular reserve capacity (CVR) in patients with a history of hypertension, coupled with the impact and cutting effect of high-pressure blood flow on the blood vessel wall, make endothelial cells damaged and prone to form microthrombus, which make hypertensive patients more likely to have postoperative stroke events. ${ }^{38}$

History of diabetes: We found that the history of diabetes is related to TND (P®0.05). Excessive blood glucose interferes with the endogenous cardiovascular nitric oxide system, resulting in the decrease of nitric oxide-dependent endothelial dilation function and the decrease of brain CVR. In addition, hyperglycemia reduces the oxygen delivery capacity of hemoglobin, aggravates brain ischemia, leads to lactate accumulation and intracellular acidosis, which in turn aggravates cerebral ischemia. ${ }^{39,40}$

History of aneurysm: The relationship between complications after revascularization and aneurysms is not clear. although patients with MMD are often associated with intracranial aneurysms, we have not observed an increase in the risk of bleeding after bypass graft. on the contrary, some scholars have also reported that aneurysms decrease or even disappear after revascularization. ${ }^{41,42}$

\section{Moyamoya disease information:}

The main clinical symptom: Previous studies have shown that postoperative CHS is independently associated with preoperative ischemic manifestations. ${ }^{28}$ However, our study did not find the relationship between preoperative main clinical symptom and TND, but we found a relationship between preoperative main clinical symptom and postoperative stroke. The incidence of postoperative stroke events in patients with cerebral infarction as the main clinical symptom before operation was higher than that with cerebral hemorrhage as the main symptom before operation $(P<0.0125)$, and 10 cases of postoperative cerebral infarction occurred in patients whose preoperative main clinical symptom was cerebral infarction. however, one of the 2 patients with postoperative cerebral hemorrhage had cerebral infarction as the main 
symptom before operation, which indicated that the patients with ischemic stroke before operation were more likely to have cerebral infarction after operation. It is consistent with the research of Park et al. ${ }^{30}$

Preoperative attack times: Multiple episodes of preoperative symptoms is a relatively clear risk factor, which has been reported by several studies. ${ }^{13,30,43}$ Multiple episodes of preoperative symptoms indicate that the compensatory ability of intracranial vessels is obviously impaired and CVR is seriously insufficient so that symptoms appear repeatedly before operation, and it is more difficult to adapt to the sudden increase of blood flow after operation.

The interval between the last attack and operation: Kim et al. ${ }^{44}$ believe that revascularization should be delayed for at least 6 weeks after the previous cerebral infarction. At the same time, some scholars believe that early surgery in children can improve intellectual outcomes. ${ }^{45}$ The center believes that patients with stroke symptoms should wait for surgical treatment after the acute stage, it is safer after one month of cerebral infarction and three months after cerebral hemorrhage, and the patients with TIA or chronic ischemia should be intervened as soon as possible. ${ }^{46}$ The stroke patients in our cohort who underwent surgical treatment in the acute stage were evaluated to recover well, and there was a selection bias, so it was not possible to observe whether the acute surgery had any effect on the complications.

Stage classification of MMD: Zhao et al. ${ }^{28}$ believe that the later the Suzuki staging of MMD, the more likely it is to have complications. We believe that some MMD patients in the later stage have formed perfect compensatory collateral vessels such as anterior choroid vessels, posterior circulation, and extracranial vessels, ${ }^{1}$ on the contrary, the degree of intracranial ischemia will be alleviated because of the powerful compensation system. Similarly, our results do not show the relationship between stage classification of MMD and complications.

Lesions involving the posterior circulation: In this study, the lesions involving the posterior circulation were considered to be related not only to TND but also to postoperative stroke. The posterior circulation is one of the most important intracranial compensatory vessels in patients with MMD. The typical MMD first involves the internal carotid artery system, the posterior communicating artery will open, and the vertebrobasilar artery can supply blood to the anterior circulation through the cerebral arterial circle. According to the Suzuki stage, at stage 4 , the cerebral arterial circle or even the posterior cerebral artery was occluded, and the vertebrobasilar artery system could only play a compensatory role through the new collateral circulation. At stage 6 , the blood supply of the brain completely depended on the collateral circulation formed by the external carotid artery and the vertebrobasilar artery system. ${ }^{5,47}$ However, in fact, not all MMD patients can successfully form sufficient posterior circulation lateral compensatory vessels. Bao et al. ${ }^{12}$ have observed that about $30 \%$ of MMD patients have posterior circulation stenosis or even occlusion. Once the posterior circulation is occluded, the narrow internal carotid artery system will lose an important source of compensatory blood vessels. In the face of sudden high flow superficial temporal artery (STA) blood flow, the already fragile intracranial blood structure may be disturbed by the new bypass vessels, and it is not difficult to understand that the incidence of postoperative complications will increase. ${ }^{30,48}$ 


\section{Surgery and Anesthesia Management:}

Operation method: In theory, the direct bypass has a faster impact on intracranial blood flow, so its perioperative complications should be higher than indirect bypass, but a Meta-analysis shows that there is no significant difference in direct and indirect bypass complications. ${ }^{49}$ The center will only use indirect bypass surgery for those whose basic physical conditions are too poor to tolerate the operation and those who cannot find a suitable recipient vessel, so our results show that the incidence of complications of the indirect bypass is slightly higher, but the difference is not statistically significant.

Operation side: The study of Hwang et al. ${ }^{50}$ suggests that left side surgery will bring more complications, probably because there are more functional areas on the left side of the brain, and the left side of the brain is more likely to show visible symptoms in the face of blood flow disorders. However, our study did not find any difference between them.

Times of bypass: We also want to explore whether patients who underwent second cerebral revascularization are more tolerant to STA blood flow than patients who have experienced only once, but the results are not different, possibly because the blood flow structures of the two hemispheres are relatively independent.

$\mathrm{PCO}_{2}$ : Under physiological conditions, intracranial vessels dilate in hypercapnia and contract in hypocapnia, but for patients with MMD, the intracranial diseased vessels have already dilated to the maximum extent, so the increase of $\mathrm{PCO}_{2}$ will only dilate the vessels without lesions, resulting in the phenomenon of blood theft. ${ }^{51}$ This effect of $\mathrm{PCO}_{2}$ is usually seen in perioperative cerebral infarction caused by crying and hyperventilation in children with MMD. ${ }^{52}$

Other factors, such as intraoperative blood loss, urine volume, and hemoglobin concentration, represent the effective circulating blood volume, which is mainly involved in the occurrence of ischemic stroke. In addition, Hwang et al. ${ }^{50}$ also found that the increase of postoperative white blood cell count in blood was related to TND. However, the significance of these factors was not found in this study.

\section{Perioperative management data:}

Blood pressure: The center has carried out strict blood pressure management for all patients with MMD, requiring all patients to maintain perioperative systolic blood pressure at $120 \mathrm{mmHg} 140 \mathrm{mmHg}$, but there are still some patients who are difficult to correct. Our study found that preoperative, intraoperative, and postoperative high blood pressure was associated with the occurrence of postoperative stroke, and postoperative high blood pressure was also associated with TND. The increase of blood pressure during and after operation will increase the perfusion pressure of donor and recipient blood vessels, and make the contradiction between the two kinds of blood flow more intense, which will not only aggravate the hyperperfusion injury, but also shift the watershed, and even trigger cerebral infarction in the contralateral hemisphere. ${ }^{32}$ In addition, severe fluctuations in blood pressure during and after operation can also have adverse effects. Fluctuating blood pressure makes hyperperfusion injury come and go, which also 
increases the difficulty of judgment. For fragile collateral circulation, their ability to automatically regulate blood flow is impaired, unable to adapt to the ups and downs of blood pressure, resulting in intracranial hemorrhage. ${ }^{33}$ Fujimura et al. ${ }^{38}$ reported that controlling systolic blood pressure at $110 \mathrm{mmHg} 130 \mathrm{mmHg}$ can reduce the risk of symptomatic hyperperfusion from $24.7 \%$ to $6.7 \%$. However, blindly reducing blood pressure can increase the risk of cerebral infarction. Therefore, in the future, large sample studies are still needed to guide the perioperative blood pressure management of MMD patients after surgery. ${ }^{38,53}$

The multivariate logistic regression analysis showed that multiple episodes of preoperative symptoms $(P=0.016)$ and lesions involving the posterior circulation $(P=0.014)$ are the independent risk factors for $T N D$, and preoperative cerebral infarction as the main clinical symptom of MMD $(P=0.015)$ is an independent risk factor for perioperative stroke in patients with MMD. When this kind of high-risk patient is encountered clinically, Indocyanine Green Video Angiography can be used to select appropriate recipient vessels during operation to reduce the occurrence of postoperative hyperperfusion syndrome. ${ }^{54-56}$ Besides, we believe that intensive blood pressure lowering therapy should not be blindly carried out after the occurrence of TND, because for TND with watershed shift, blindly lowering blood pressure may lead to ischemic stroke at the watershed. ${ }^{57}$ On the contrary, for patients with new cerebral infarction caused by a watershed shift, blindly expanding blood volume and increased blood pressure may induce the emergence of a high perfusion area. It is suggested that the postoperative cerebral blood flow of high-risk patients should be measured and the treatment plan should be decided after comprehensive consideration. ${ }^{58}$ Moreover, after cerebral revascularization in patients with MMD, a large amount of free radical will be produced due to ischemia-reperfusion injury, which will aggravate the damage caused by hyperperfusion. Edaravone ${ }^{59}$, a free radical scavenger, and minocycline ${ }^{60}$, an inhibitor of matrix metalloproteinase 9 (MMP-9), have been proved to be effective on perfusion disorders after cerebral vascular revascularization.

\section{Follow-up outcomes:}

All patients were followed up for more than 3 months (Md=15 months, IQR= 9 months). Meanwhile, mRS scores and cerebral rehemorrhage were collected during follow-up. Comparing the improvement of mRS score between the group with and without postoperative complications, it was found that there was no statistical difference between the two groups, which was consistent with the results of Zhao et al. ${ }^{28}$ It may be because the patients with stroke events were treated at the first time, while the patients with TND did not leave neurological dysfunction in the long term. During the follow-up, we found that 8 patients had cerebral rehemorrhage, all of which occurred in patients without perioperative complications, suggesting that the pathogenesis of perioperative stroke may be different from that of long-term stroke in patients with MMD.

\section{Limitations:}

Finally, this study has some limitations: (1) All 183 patients included in this study were from a single center with geographical restrictions. (2) The blood pressure and $\mathrm{PCO}_{2}$ levels observed in the study are not 
the results of continuous monitoring and cannot be well used to represent the whole perioperative period.

(3) A portion of the postoperative stroke cannot clearly judge the time relationship between blood pressure changes and stroke. There may be a situation that reactive blood pressure rises after stroke. we do not collect blood pressure information after complications, as far as possible to avoid the occurrence of this kind of error.

\section{Conclusion}

Our study found that multiple episodes of preoperative symptoms and lesions involving the posterior circulation were independent risk factors for postoperative TND, and preoperative cerebral infarction as the main clinical symptom of MMD was an independent risk factor for postoperative stroke. During the follow-up, it was found that there was no correlation between the occurrence of postoperative complications and the improvement of long-term prognosis and long-term cerebral rehemorrhage.

\section{Declarations}

\section{Conflict of interest statement}

We declare that we do not have any commercial or associative interest that represents a conflict of interest in connection with the work submitted. The author have no relevant financial or non-financial interests to disclose.

\section{Ethics approval and consent to participate}

The study was carried out in accordance with The Code of Ethics of the World Medical Association (Declaration of Helsinki) for experiments involving humans, and the protocol was approved by the Ethics Committee of Tongji hospital of Tongji medical college of Huazhong University of Science and Technology. Informed consent was obtained for experimentation with human subjects. Every individual was informed about the aims of the study and provided written consent before participation. Written informed consent was obtained from a relative in the case of patients with impaired cognition.

\section{Consent for publication}

Not applicable.

\section{Availability of data and materials}

All data generated or analyzed during this study are included in this published article. Patient-level data will be available on request, provided that the Regional Ethics Committee give approval.

\section{Fundings}

This work is supported by the Natural Science Foundation of Hubei province (WJ2019Z008). The Natural Science Foundation of Tongji Hospital(2020JZKT651). 
$\mathrm{HZ}$, TL, KS, SW and CG designed and supervised the project; XZ collected data, carried out data analysis, part of the follow-up work and paper writing; $Y Y$ participated in some statistical analysis and follow-up; $\mathrm{XH}$ was responsible for the collection of clinical data.; $\mathrm{YH}$ and $\mathrm{YL}$ was responsible for data analysis and revised the manuscript; XM participated in data collection and follow-up. All authors approved the final version of the paper.

\section{Acknowledgments}

This work is supported by the Natural Science Foundation of Hubei province (WJ2019Z008). The Natural Science Foundation of Tongji Hospital(2020JZKT651).

\section{References}

1. Suzuki J, Takaku A. Cerebrovascular "moyamoya" disease. Disease showing abnormal net-like vessels in base of brain. Archives of neurology. 1969;20(3): 288-299. https://doi.org/10.1001/archneur.1969.00480090076012.

2. Sun Y, Zhou G, Feng J, et al. Incidence and prevalence of moyamoya disease in urban China: a nationwide retrospective cohort study. Stroke and vascular neurology. 2021. https://doi.org/10.1136/svn-2021-000909.

3. Bao X, Wang Q, Zhang Y, et al. Epidemiology of Moyamoya Disease in China: Single-Center, Population-Based Study. World neurosurgery. 2019;122: e917-e923. https://doi.org/10.1016/j.wneu.2018.10.175.

4. Pandey P, Steinberg G. Neurosurgical advances in the treatment of moyamoya disease. Stroke. 2011;42(11): 3304-3310. https://doi.org/10.1161/strokeaha.110.598565.

5. Guidelines for diagnosis and treatment of moyamoya disease (spontaneous occlusion of the circle of Willis). Neurologia medico-chirurgica. 2012;52(5): 245-266. https://doi.org/10.2176/nmc.52.245.

6. Li Q, Gao Y, Xin W, et al. Meta-Analysis of Prognosis of Different Treatments for Symptomatic Moyamoya Disease. World neurosurgery. 2019;127: 354-361. https://doi.org/10.1016/j.wneu.2019.04.062.

7. Fujimura M, Shimizu H, Inoue T, Mugikura S, Saito A, Tominaga T. Significance of focal cerebral hyperperfusion as a cause of transient neurologic deterioration after extracranial-intracranial bypass for moyamoya disease: comparative study with non-moyamoya patients using $\mathrm{N}$-isopropyl-p[(123)I]iodoamphetamine single-photon emission computed tomography. Neurosurgery. 2011;68(4): 957-964; discussion 964-955. https://doi.org/10.1227/NEU.0b013e318208f1da.

8. Yu J, Zhang J, Li J, Zhang J, Chen J. Cerebral Hyperperfusion Syndrome After Revascularization Surgery in Patients with Moyamoya Disease: Systematic Review and Meta-Analysis. World neurosurgery. 2020;135: 357-366.e354. https://doi.org/10.1016/j.wneu.2019.11.065. 
9. Kuriyama S, Kusaka Y, Fujimura M, et al. Prevalence and clinicoepidemiological features of moyamoya disease in Japan: findings from a nationwide epidemiological survey. Stroke. 2008;39(1): 42-47. https://doi.org/10.1161/strokeaha.107.490714.

10. Im S, Yim S, Cho C, et al. Prevalence and epidemiological features of moyamoya disease in Korea. Journal of cerebrovascular and endovascular neurosurgery. 2012;14(2): 75-78. https://doi.org/10.7461/jcen.2012.14.2.75.

11. Liu X, Zhang D, Wang S, et al. Clinical features and long-term outcomes of moyamoya disease: a single-center experience with 528 cases in China. Journal of neurosurgery. 2015;122(2): 392-399. https://doi.org/10.3171/2014.10.Jns132369.

12. Bao X, Duan L, Li D, et al. Clinical features, surgical treatment and long-term outcome in adult patients with Moyamoya disease in China. Cerebrovascular diseases (Basel, Switzerland). 2012;34(4): 305313. https://doi.org/10.1159/000343225.

13. Zhai X, Mao L, Wang H, et al. Risk Factors Associated with Neurologic Deterioration After Combined Direct and Indirect Revascularization in Patients with Moyamoya Disease on the East Coast of China. World neurosurgery. 2018;118: e92-e98. https://doi.org/10.1016/j.wneu.2018.06.125.

14. Kim J, Oh C, Kwon O, Park S, Kim S, Kim Y. Transient hyperperfusion after superficial temporal artery/middle cerebral artery bypass surgery as a possible cause of postoperative transient neurological deterioration. Cerebrovascular diseases (Basel, Switzerland). 2008;25(6): 580-586. https://doi.org/10.1159/000132205.

15. Ohue S, Kumon $\mathrm{Y}$, Kohno K, Watanabe H, Iwata S, Ohnishi T. Postoperative temporary neurological deficits in adults with moyamoya disease. Surgical neurology. 2008;69(3): 281-286; discussion 286287. https://doi.org/10.1016/j.surneu.2007.01.047.

16. Fujimura M, Kaneta T, Mugikura S, Shimizu H, Tominaga T. Temporary neurologic deterioration due to cerebral hyperperfusion after superficial temporal artery-middle cerebral artery anastomosis in patients with adult-onset moyamoya disease. Surgical neurology. 2007;67(3): 273-282. https://doi.org/10.1016/j.surneu.2006.07.017.

17. Fujimura M, Tominaga T. Lessons learned from moyamoya disease: outcome of direct/indirect revascularization surgery for 150 affected hemispheres. Neurologia medico-chirurgica. 2012;52(5): 327-332. https://doi.org/10.2176/nmc.52.327.

18. Iwama T, Hashimoto N, Tsukahara T, Murai B. Peri-operative complications in adult moyamoya disease. Acta neurochirurgica. 1995;132: 26-31. https://doi.org/10.1007/bf01404844.

19. Uno M, Nakajima N, Nishi K, Shinno K, Nagahiro S. Hyperperfusion syndrome after extracranialintracranial bypass in a patient with moyamoya disease--case report. Neurologia medico-chirurgica. 1998;38(7): 420-424. https://doi.org/10.2176/nmc.38.420.

20. Hayashi T, Shirane R, Fujimura M, Tominaga T. Postoperative neurological deterioration in pediatric moyamoya disease: watershed shift and hyperperfusion. Journal of neurosurgery Pediatrics. 2010;6(1): 73-81. https://doi.org/10.3171/2010.4.Peds09478. 
21. Yu J, Hu M, Yi L, Zhou K, Zhang J, Chen J. Paradoxical association of symptomatic cerebral edema with local hypoperfusion caused by the 'watershed shift' after revascularization surgery for adult moyamoya disease: a case report. Therapeutic advances in neurological disorders. 2019;12: 1756286419878343. https://doi.org/10.1177/1756286419878343.

22. Tashiro R, Fujimura M, Kameyama M, et al. Incidence and Risk Factors of the Watershed Shift Phenomenon after Superficial Temporal Artery-Middle Cerebral Artery Anastomosis for Adult Moyamoya Disease. Cerebrovascular diseases (Basel, Switzerland). 2019;47: 178-187. https://doi.org/10.1159/000500802.

23. Mukerji N, Cook D, Steinberg G. Is local hypoperfusion the reason for transient neurological deficits after STA-MCA bypass for moyamoya disease? Journal of neurosurgery. 2015;122(1): 90-94. https://doi.org/10.3171/2014.8.Jns132413.

24. Phi J, Lee S, Kang H, et al. Postoperative Transient Neurologic Dysfunction: A Proposal for Pathophysiology. Journal of clinical neurology (Seoul, Korea). 2018;14(3): 393-400. https://doi.org/10.3988/jcn.2018.14.3.393.

25. Ishii D, Okazaki T, Matsushige T, et al. Postoperative Dilatation of Superficial Temporal Artery Associated with Transient Neurologic Symptoms After Direct Bypass Surgery for Moyamoya Angiopathy. World neurosurgery. 2017;106: 435-441. https://doi.org/10.1016/j.wneu.2017.07.037.

26. Zhang M, Tang J, Liu N, Xue Y, Ren X, Fu J. Postoperative Functional Outcomes and Prognostic Factors in Two Types of Adult Moyamoya Diseases. Journal of stroke and cerebrovascular diseases : the official journal of National Stroke Association. 2020;29(8): 104846. https://doi.org/10.1016/j.jstrokecerebrovasdis.2020.104846.

27. Kazumata $\mathrm{K}$, Ito $\mathrm{M}$, Tokairin $\mathrm{K}$, et al. The frequency of postoperative stroke in moyamoya disease following combined revascularization: a single-university series and systematic review. Journal of neurosurgery. 2014;121(2): 432-440. https://doi.org/10.3171/2014.1.Jns13946.

28. Zhao $M$, Deng $X$, Zhang $D$, et al. Risk factors for and outcomes of postoperative complications in adult patients with moyamoya disease. J Neurosurg. 2018: 1-12. https://doi.org/10.3171/2017.10.Jns171749.

29. Muraoka S, Araki Y, Kondo G, et al. Postoperative Cerebral Infarction Risk Factors and Postoperative Management of Pediatric Patients with Moyamoya Disease. World neurosurgery. 2018;113: e190e199. https://doi.org/10.1016/j.wneu.2018.01.212.

30. Park W, Ahn J, Lee H, Park J, Kwun B. Risk Factors for Newly Developed Cerebral Infarction After Surgical Revascularization for Adults with Moyamoya Disease. World neurosurgery. 2016;92: 65-73. https://doi.org/10.1016/j.wneu.2016.03.053.

31. Fujimura M, Kaneta T, Shimizu $H$, Tominaga T. Cerebral ischemia owing to compression of the brain by swollen temporal muscle used for encephalo-myo-synangiosis in moyamoya disease. Neurosurgical review. 2009;32(2): 245-249; discussion 249. https://doi.org/10.1007/s10143-0090184-6. 
32. Sussman E, Madhugiri V, Teo M, et al. Contralateral acute vascular occlusion following revascularization surgery for moyamoya disease. Journal of neurosurgery. 2018;131(6): 1702-1708. https://doi.org/10.3171/2018.8.Jns18951.

33. Tokairin K, Kazumata K, Uchino H, et al. Postoperative Intracerebral Hemorrhage After Combined Revascularization Surgery in Moyamoya Disease: Profiles and Clinical Associations. World neurosurgery. 2018;120: e593-e600. https://doi.org/10.1016/j.wneu.2018.08.132.

34. Chen Y, Ma L, Lu J, et al. Postoperative hemorrhage during the acute phase after direct or combined revascularization for moyamoya disease: risk factors, prognosis, and literature review. Journal of neurosurgery. 2019: 1-10. https://doi.org/10.3171/2019.7.Jns19885.

35. Park W, Park E, Lee S, et al. Intracranial Hemorrhage After Superficial Temporal Artery-Middle Cerebral Artery Direct Anastomosis for Adults with Moyamoya Disease. World neurosurgery. 2018;119: e774e782. https://doi.org/10.1016/j.wneu.2018.07.266.

36. Kazumata K, Tha K, Uchino H, et al. Topographic changes in cerebral blood flow and reduced white matter integrity in the first 2 weeks following revascularization surgery in adult moyamoya disease. Journal of neurosurgery. 2017;127(2): 260-269. https://doi.org/10.3171/2016.6.Jns16653.

37. Fujimura M, Shimizu H, Mugikura S, Tominaga T. Delayed intracerebral hemorrhage after superficial temporal artery-middle cerebral artery anastomosis in a patient with moyamoya disease: possible involvement of cerebral hyperperfusion and increased vascular permeability. Surgical neurology. 2009;71(2): 223-227; discussion 227. https://doi.org/10.1016/j.surneu.2007.07.077.

38. Fujimura M, Inoue T, Shimizu H, Saito A, Mugikura S, Tominaga T. Efficacy of prophylactic blood pressure lowering according to a standardized postoperative management protocol to prevent symptomatic cerebral hyperperfusion after direct revascularization surgery for moyamoya disease. Cerebrovascular diseases (Basel, Switzerland). 2012;33(5): 436-445. https://doi.org/10.1159/000336765.

39. Zhao M, Deng X, Gao F, et al. Ischemic Stroke in Young Adults with Moyamoya Disease: Prognostic Factors for Stroke Recurrence and Functional Outcome after Revascularization. World neurosurgery. 2017;103: 161-167. https://doi.org/10.1016/j.wneu.2017.03.146.

40. Shou J, Zhou L, Zhu S, Zhang X. Diabetes is an Independent Risk Factor for Stroke Recurrence in Stroke Patients: A Meta-analysis. Journal of stroke and cerebrovascular diseases : the official journal of National Stroke Association. 2015;24(9): 1961-1968. https://doi.org/10.1016/j.jstrokecerebrovasdis.2015.04.004.

41. Ni W, Jiang $H, X u B$, et al. Treatment of aneurysms in patients with moyamoya disease: a 10-year single-center experience. Journal of neurosurgery. 2018;128(6): 1813-1822. https://doi.org/10.3171/2017.3.Jns162290.

42. Liu P, Lv X, Liu A, et al. Intracranial Aneurysms Associated with Moyamoya Disease in Children: Clinical Features and Long-Term Surgical Outcome. World neurosurgery. 2016;94: 513-520. https://doi.org/10.1016/j.wneu.2016.05.039. 
43. Hyun S, Kim J, Hong S. Prognostic factors associated with perioperative ischemic complications in adult-onset moyamoya disease. Acta neurochirurgica. 2010;152(7): 1181-1188. https://doi.org/10.1007/s00701-010-0638-1.

44. Kim S, Choi J, Yang K, Kim T, Kim D. Risk factors for postoperative ischemic complications in patients with moyamoya disease. Journal of neurosurgery. 2005;103: 433-438. https://doi.org/10.3171/ped.2005.103.5.0433.

45. Ishii R, Takeuchi S, Ibayashi K, Tanaka R. Intelligence in children with moyamoya disease: evaluation after surgical treatments with special reference to changes in cerebral blood flow. Stroke. 1984;15(5): 873-877. https://doi.org/10.1161/01.str.15.5.873.

46. Xu S, Zhang J, Wang S, et al. The Optimum Operative Time of Revascularization for Patients with Moyamoya Disease Following Acute Onset. World neurosurgery. 2018;114: e412-e416. https://doi.org/10.1016/j.wneu.2018.02.193.

47. Miyamoto S, Kikuchi H, Karasawa J, Nagata I, Ikota T, Takeuchi S. Study of the posterior circulation in moyamoya disease. Clinical and neuroradiological evaluation. J Neurosurg. 1984;61(6): 1032-1037. https://doi.org/10.3171/jns.1984.61.6.1032.

48. Yu L, Ma L, Huang Z, et al. Revascularization Surgery in Patients with Ischemic-Type Moyamoya Disease: Predictors for Postoperative Stroke and Long-Term Outcomes. World Neurosurg. 2019;128: e582-e596. https://doi.org/10.1016/j.wneu.2019.04.214.

49. Jeon JP, Kim JE, Cho WS, Bang JS, Son YJ, Oh CW. Meta-analysis of the surgical outcomes of symptomatic moyamoya disease in adults. J Neurosurg. 2018;128(3): 793-799. https://doi.org/10.3171/2016.11.Jns161688.

50. Hwang JW, Yang HM, Lee $\mathrm{H}$, et al. Predictive factors of symptomatic cerebral hyperperfusion after superficial temporal artery-middle cerebral artery anastomosis in adult patients with moyamoya disease. Br J Anaesth. 2013;110(5): 773-779. https://doi.org/10.1093/bja/aes470.

51. Oshima H, Katayama $\mathrm{Y}$, Hirayama T. Intracerebral steal phenomenon associated with global hyperemia in moyamoya disease during revascularization surgery. J Neurosurg. 2000;92(6): 949-954. https://doi.org/10.3171/jns.2000.92.6.0949.

52. Sakamoto T, Kawaguchi M, Kurehara K, Kitaguchi K, Furuya H, Karasawa J. Postoperative neurological deterioration following the revascularization surgery in children with moyamoya disease. J Neurosurg Anesthesiol. 1998;10(1): 37-41. https://doi.org/10.1097/00008506-199801000-00009.

53. Li C, Zhang N, Yu S, et al. Individualized Perioperative Blood Pressure Management for Adult Moyamoya Disease: Experience from 186 Consecutive Procedures. J Stroke Cerebrovasc Dis. 2021;30(1): 105413. https://doi.org/10.1016/j.jstrokecerebrovasdis.2020.105413.

54. Saito M, Saga T, Hayashi H, Noro S, Wada H, Kamada K. Quantitative Blood Flow Assessment by Multiparameter Analysis of Indocyanine Green Video Angiography. World Neurosurg. 2018;116: e187e193. https://doi.org/10.1016/j.wneu.2018.04.148.

55. Zhang X, Ni W, Feng R, et al. Evaluation of Hemodynamic Change by Indocyanine Green-FLOW 800 Videoangiography Mapping: Prediction of Hyperperfusion Syndrome in Patients with Moyamoya 
Disease. Oxid Med Cel/ Longev. 2020;2020: 8561609. https://doi.org/10.1155/2020/8561609.

56. Rennert RC, Strickland BA, Ravina K, Bakhsheshian J, Russin JJ. Assessment of Hemodynamic Changes and Hyperperfusion Risk After Extracranial-to-Intracranial Bypass Surgery Using Intraoperative Indocyanine Green-Based Flow Analysis. World Neurosurg. 2018;114: 352-360. https://doi.org/10.1016/j.wneu.2018.03.189.

57. Tu XK, Fujimura M, Rashad S, et al. Uneven cerebral hemodynamic change as a cause of neurological deterioration in the acute stage after direct revascularization for moyamoya disease: cerebral hyperperfusion and remote ischemia caused by the 'watershed shift'. Neurosurg Rev. 2017;40(3): 507512. https://doi.org/10.1007/s10143-017-0845-9.

58. Kameyama M, Fujimura M, Tashiro R, et al. Significance of Quantitative Cerebral Blood Flow Measurement in the Acute Stage after Revascularization Surgery for Adult Moyamoya Disease: Implication for the Pathological Threshold of Local Cerebral Hyperperfusion. Cerebrovasc Dis. 2019;48(3-6): 217-225. https://doi.org/10.1159/000504835.

59. Uchino H, Nakayama N, Kazumata K, Kuroda S, Houkin K. Edaravone Reduces HyperperfusionRelated Neurological Deficits in Adult Moyamoya Disease: Historical Control Study. Stroke. 2016;47(7): 1930-1932. https://doi.org/10.1161/strokeaha.116.013304.

60. Fujimura M, Niizuma K, Inoue T, et al. Minocycline prevents focal neurological deterioration due to cerebral hyperperfusion after extracranial-intracranial bypass for moyamoya disease. Neurosurgery. 2014;74(2): 163-170; discussion 170. https://doi.org/10.1227/neu.0000000000000238.

\section{Tables}

Table1. Baseline Characteristics of 203 cases of revascularization of MMD 


\begin{tabular}{|c|c|}
\hline Characteristics & $\mathrm{n}(\%)$ \\
\hline \multicolumn{2}{|l|}{ Gender } \\
\hline Male & $110(54.2)$ \\
\hline Female & $93(45.8)$ \\
\hline Age (, years) & $46.76 \pm 11.15$ \\
\hline \multicolumn{2}{|l|}{ History } \\
\hline Hypertension & $72(35.5)$ \\
\hline Diabetes & $25(12.3)$ \\
\hline Aneurysm & $22(10.8)$ \\
\hline \multicolumn{2}{|l|}{ Moyamoya disease information } \\
\hline \multicolumn{2}{|l|}{ The main clinical symptom } \\
\hline Cerebral hemorrhage & $92(45.3)$ \\
\hline Cerebral infarction & $54(26.6)$ \\
\hline TIA & $31(15.3)$ \\
\hline Chronic cerebral ischemia & $26(12.8)$ \\
\hline \multicolumn{2}{|l|}{ Preoperative attack times } \\
\hline One time & $160(78.8)$ \\
\hline Two times and more & $43(21.2)$ \\
\hline \multicolumn{2}{|c|}{ Interval between the last attack and operation } \\
\hline Acute operation & $41(20.2)$ \\
\hline Stable operation & $94(46.3)$ \\
\hline Long-term operation & $68(33.5)$ \\
\hline \multicolumn{2}{|l|}{ Stage classification } \\
\hline Early stage & $16(7.9)$ \\
\hline Middle stage & $142(70)$ \\
\hline Late stage & $45(22.2)$ \\
\hline Lesions involving the posterior circulation & $54(26.6)$ \\
\hline Admission mRS score (, marks) & $1.82 \pm 1.02$ \\
\hline Follow-up mRS score (, marks) & $1.34 \pm 1.18$ \\
\hline
\end{tabular}


Table2. Surgical information of $\mathbf{2 0 3}$ cases of revascularization of MMD

\begin{tabular}{|c|c|}
\hline Information & n (\%) \\
\hline \multicolumn{2}{|l|}{ Operation method } \\
\hline EDMS + STA-MCA bypass & $196(96.6)$ \\
\hline EDMS & $7(3.4)$ \\
\hline \multicolumn{2}{|l|}{ Operation side } \\
\hline Left & $95(46.8)$ \\
\hline Right & $108(53.2)$ \\
\hline \multicolumn{2}{|l|}{ Times of bypass } \\
\hline The first time & $172(84.7)$ \\
\hline The second time & $31(15.3)$ \\
\hline Duration of surgery [(Md, IQR), minute] & $218(71)$ \\
\hline Intraoperative blood loss (, mL) & $138.77 \pm 129.76$ \\
\hline \multicolumn{2}{|l|}{ Intraoperative $\mathrm{PCO}_{2}$} \\
\hline Low & $40(19.7)$ \\
\hline Normal & $161(79.3)$ \\
\hline High & $2(1)$ \\
\hline \multicolumn{2}{|l|}{ Perioperative blood pressure } \\
\hline \multicolumn{2}{|l|}{ Preoperative } \\
\hline Normal & $179(88.2)$ \\
\hline High & $24(11.8)$ \\
\hline \multicolumn{2}{|l|}{ Intraoperative } \\
\hline Normal & $186(91.6)$ \\
\hline High & $17(8.4)$ \\
\hline \multicolumn{2}{|l|}{ Postoperative } \\
\hline Normal & $161(79.3)$ \\
\hline High & $42(20.7)$ \\
\hline $24 \mathrm{~h}$ cranial drainage volume after operation $(, \mathrm{mL})$ & $161.63 \pm 81.09$ \\
\hline
\end{tabular}




\begin{tabular}{|lll|}
\hline Complications & Total (case) & $\mathbf{n}(\%)$ \\
\hline Perioperative period & \\
\hline TND & $26(12.8 \%)$ & \\
\hline Aphasia & $7(26.9)$ \\
\hline Epilepsy & $4(15.4)$ \\
\hline Muscle weakness + Aphasia & $3(11.5)$ \\
\hline Severe vomiting + Vertigo & $3(11.5)$ \\
\hline Muscle weakness & $2(7.7)$ \\
\hline Central facial paralysis & $2(7.7)$ \\
\hline Consciousness disorders & $2(7.7)$ \\
\hline Acroparesthesia & $1(3.8)$ \\
\hline Blurred vision & $1(3.8)$ \\
\hline Drinking water cough + Epilepsy & $1(3.8)$ \\
\hline Stroke & \\
\hline Infarction & & \\
\hline Hemorrhage & & \\
\hline Long term & & \\
\hline Cerebral rebleeding & & \\
\hline
\end{tabular}

Table4. The univariate analysis of perioperative complications after revascularization in 203 operations 


\begin{tabular}{|c|c|c|c|c|c|c|}
\hline \multirow[t]{3}{*}{ Factors } & \multicolumn{3}{|l|}{ TND } & \multicolumn{3}{|c|}{ Perioperative Stroke } \\
\hline & TND (+) & TND (-) & $p$ & Stroke $(+)$ & Stroke (-) & $p$ \\
\hline & $n(\%)(n=26)$ & $\begin{array}{l}n(\%) \\
(n=177)\end{array}$ & & $n(\%)(n=12)$ & $\begin{array}{l}n(\%) \\
(n=191)\end{array}$ & \\
\hline $\begin{array}{l}\text { Gender } \\
\text { (male/female) } \\
+\end{array}$ & $13 / 13$ & $97 / 80$ & 0.646 & $8 / 4$ & $102 / 89$ & 0.371 \\
\hline Age $(, \text { years })^{\ddagger}$ & $43.2 \pm 12.9$ & $47.3 \pm 10.8$ & 0.081 & $56 \pm 8.7$ & $46.2 \pm 11.1$ & $0.003^{*}$ \\
\hline \multicolumn{7}{|l|}{ History $^{\dagger}$} \\
\hline Hypertension & $7(26.9)$ & $65(36.7)$ & 0.329 & $8(66.7)$ & $64(33.5)$ & $0.044^{*}$ \\
\hline Diabetes & $7(26.9)$ & $18(10.2)$ & $0.035^{*}$ & $4(33.3)$ & $21(11)$ & 0.067 \\
\hline Aneurysm & $2(7.7)$ & $20(11.3)$ & 0.83 & $3(25)$ & $19(9.9)$ & 0.251 \\
\hline $\begin{array}{l}\text { The main } \\
\text { clinical } \\
\text { symptom }\end{array}$ & & & 0.328 & & & $0.001^{*}$ \\
\hline $\begin{array}{l}\text { Cerebral } \\
\text { hemorrhage }\end{array}$ & $9(34.6)$ & $83(46.9)$ & & $1(8.3)$ & $91(47.6)$ & \\
\hline $\begin{array}{l}\text { Cerebral } \\
\text { infarction }\end{array}$ & 7 (26.9) & $47(26.6)$ & & $11(91.7)$ & $43(22.5)$ & \\
\hline TIA & 7 (26.9) & $24(13.6)$ & & $0(0)$ & $31(16.2)$ & \\
\hline $\begin{array}{l}\text { Chronic } \\
\text { cerebral } \\
\text { ischemia }\end{array}$ & $3(11.5)$ & $23(13)$ & & $0(0)$ & $26(13.6)$ & \\
\hline $\begin{array}{l}\text { Preoperative } \\
\text { attack times }^{\dagger}\end{array}$ & & & $0.005^{*}$ & & & 0.976 \\
\hline One time & $15(57.7)$ & 145 (81.9) & & 10 (83.3) & $150(78.5)$ & \\
\hline $\begin{array}{l}\text { Two times } \\
\text { and more }\end{array}$ & $11(42.3)$ & $32(18.1)$ & & $2(16.7)$ & $41(21.5)$ & \\
\hline $\begin{array}{l}\text { Interval } \\
\text { between the } \\
\text { last attack } \\
\text { and operation } \\
\S\end{array}$ & & & 0.876 & & & 0.237 \\
\hline $\begin{array}{l}\text { Acute } \\
\text { operation }\end{array}$ & $5(19.2)$ & $36(20.3)$ & & $2(16.7)$ & $39(20.4)$ & \\
\hline $\begin{array}{l}\text { Stable } \\
\text { operation }\end{array}$ & $12(46.2)$ & $82(46.3)$ & & $9(75)$ & $85(44.5)$ & \\
\hline
\end{tabular}




\begin{tabular}{|c|c|c|c|c|c|c|}
\hline $\begin{array}{l}\text { Long-term } \\
\text { operation }\end{array}$ & $9(34.6)$ & 59 (33.3) & & $1(8.3)$ & $67(35.1)$ & \\
\hline $\begin{array}{l}\text { Stage } \\
\text { classification } \\
\S\end{array}$ & & & 0.086 & & & 0.064 \\
\hline Early stage & $1(3.8)$ & $15(8.5)$ & & $0(0)$ & $16(8.4)$ & \\
\hline Middle stage & $16(61.5)$ & $126(71.2)$ & & $7(58.3)$ & 135 (70.7) & \\
\hline Late stage & $9(34.6)$ & $36(20.3)$ & & $5(41.7)$ & $40(20.9)$ & \\
\hline $\begin{array}{l}\text { Lesions } \\
\text { involving the } \\
\text { posterior } \\
\text { circulation }{ }^{\dagger}\end{array}$ & $13(50)$ & $41(23.2)$ & $0.004^{*}$ & $7(58.3)$ & $47(24.6)$ & $0.026^{*}$ \\
\hline $\begin{array}{l}\text { Admission } \\
\text { mRS score } \S\end{array}$ & & & 0.136 & & & 0.073 \\
\hline $0 \sim 1$ & $13(50)$ & $62(35)$ & & $2(16.7)$ & 73 (38.2) & \\
\hline $2 \sim 3$ & $12(46.2)$ & 103 (58.2) & & $8(66.7)$ & $107(56)$ & \\
\hline$\geq 4$ & $1(3.8)$ & $12(6.8)$ & & $2(16.7)$ & $11(5.8)$ & \\
\hline $\begin{array}{l}\text { Operation } \\
\text { method " }\end{array}$ & & & 0.221 & & & 1 \\
\hline $\begin{array}{l}\text { EDMS+STA- } \\
\text { MCA bypass }\end{array}$ & $24(92.3)$ & $172(97.2)$ & & $12(100)$ & $184(96.3)$ & \\
\hline EDMS & $2(7.7)$ & $5(2.8)$ & & $0(0)$ & 7 (3.7) & \\
\hline$\underset{\dagger}{\text { Operation side }}$ & & & 0.726 & & & 0.819 \\
\hline Left & $13(50)$ & $82(46.3)$ & & $6(50)$ & $89(46.6)$ & \\
\hline Right & $13(50)$ & 95 (53.7) & & $6(50)$ & $102(53.4)$ & \\
\hline $\begin{array}{l}\text { Times of } \\
\text { bypass }^{+}\end{array}$ & & & 0.784 & & & 0.27 \\
\hline The first time & $23(88.5)$ & 149 (84.2) & & $12(100)$ & 160 (83.3) & \\
\hline $\begin{array}{l}\text { The second } \\
\text { time }\end{array}$ & $3(11.5)$ & $28(15.8)$ & & $0(0)$ & $31(16.2)$ & \\
\hline $\begin{array}{l}\text { Duration of } \\
\text { surgery [(Md, } \\
\text { IQR), minute] } \S\end{array}$ & 232 (65) & $243(77)$ & 0.179 & 232 (101) & 217 (64) & 0.201 \\
\hline $\begin{array}{l}\text { Intraoperative } \\
\text { blood loss (, }\end{array}$ & $126.9 \pm 145.8$ & $140.5 \pm 127.6$ & 0.399 & $162.5 \pm 143.2$ & $137.3 \pm 129.1$ & 0.406 \\
\hline
\end{tabular}




\begin{tabular}{|c|c|c|c|c|c|c|}
\hline $\begin{array}{l}\text { Intraoperative } \\
\mathrm{PCO}_{2} \S\end{array}$ & & & 0.115 & & & 0.596 \\
\hline$\varangle 35 \mathrm{mmHg}$ & $8(30.8)$ & $32(18.1)$ & & $3(25)$ & $37(19.4)$ & \\
\hline $35 \sim 45 \mathrm{mmHg}$ & $18(69.2)$ & $143(80.8)$ & & $9(75)$ & $152(79.6)$ & \\
\hline$\nabla 45 \mathrm{mmHg}$ & $0(0)$ & $2(1.1)$ & & $0(0)$ & $2(1)$ & \\
\hline \multicolumn{7}{|l|}{$\begin{array}{l}\text { Perioperative } \\
\text { blood } \\
\text { pressure }^{+}\end{array}$} \\
\hline $\begin{array}{l}\text { High } \\
\text { preoperative } \\
\text { blood } \\
\text { pressure }\end{array}$ & $6(23.1)$ & $18(10.2)$ & 0.115 & $5(41.7)$ & $19(9.9)$ & $0.005^{\star}$ \\
\hline $\begin{array}{l}\text { High } \\
\text { intraoperative } \\
\text { blood } \\
\text { pressure }\end{array}$ & $3(11.5)$ & $14(7.9)$ & 0.807 & $4(33.3)$ & $13(6.8)$ & $0.007^{*}$ \\
\hline $\begin{array}{l}\text { High } \\
\text { postoperative } \\
\text { blood } \\
\text { pressure }\end{array}$ & $11(42.3)$ & $31(17.5)$ & $0.004^{*}$ & $8(66.7)$ & $34(17.8)$ & $0.001^{*}$ \\
\hline $\begin{array}{l}\text { 24h cranial } \\
\text { drainage } \\
\text { volume after } \\
\text { operation (, } \\
\mathrm{mL})^{\ddagger}\end{array}$ & $161.9 \pm 77.4$ & $161.6 \pm 81.8$ & 0.984 & $165 \pm 65.9$ & $161.4 \pm 82.1$ & 0.882 \\
\hline
\end{tabular}

† Chi-square test was used.

$\ddagger t$-test was used.

$\S$ rank-sum test was used.

II Fisher's exact test was used.

* $P<0.05$

Table5. The multivariate analysis of perioperative complications after revascularization in 203 operations 


\begin{tabular}{|lllll|}
\hline Risk Factors & B value & OR & $95 \% \mathrm{Cl}$ & $P$ value \\
\hline TND & & & & \\
\hline History of diabetes & -0.806 & 0.446 & $0.142 \sim 1.401$ & 0.167 \\
\hline Multiple episodes of preoperative symptoms & 1.142 & 3.134 & $1.236 \sim 7.943$ & $0.016^{*}$ \\
\hline Lesions involving the posterior circulation & -1.119 & 0.327 & $0.133 \sim 0.8$ & $0.014^{*}$ \\
\hline High postoperative blood pressure & 0.831 & 2.296 & $0.868 \sim 6.077$ & 0.094 \\
\hline Perioperative stroke & & & & \\
\hline Older age & 0.06 & 1.061 & $0.988 \sim 1.141$ & 0.104 \\
\hline History of hypertension & -0.048 & 0.953 & $0.146 \sim 6.229$ & 0.96 \\
\hline The main clinical symptom & -1.565 & 0.209 & $0.059 \sim 0.738$ & $0.015^{*}$ \\
\hline Lesions involving the posterior circulation & -1.353 & 0.258 & $0.062 \sim 1.079$ & 0.064 \\
\hline Perioperative blood pressure & & & & \\
\hline High preoperative blood pressure & -0.229 & 0.795 & $0.106 \sim 5.942$ & 0.823 \\
\hline High intraoperative blood pressure & 0.804 & 2.235 & $0.351 \sim 14.237$ & 0.395 \\
\hline High postoperative blood pressure & 1.074 & 2.928 & $0.585 \sim 14.645$ & 0.191 \\
\hline
\end{tabular}

$B$ value: partial regression coefficient.

OR: odds ratio.

95\% Cl: $95 \%$ confidence interval of odds ratio.

* $P<0.05$.

Table6. Effect of perioperative complications on long-term prognosis

\begin{tabular}{|lll|}
\hline \multicolumn{3}{|c|}{ Changes in long-term prognosis } \\
\hline & Improvement of prognosis ${ }^{9} \quad$ Deterioration of prognosis * \\
\hline Perioperative complications & & \\
\hline Occurred & $30(78.9)$ & $8(21.1)$ \\
\hline Un-occurred & $149(90.3)$ & $16(9.7)$ \\
\hline$P$ value & 0.094 & \\
\hline
\end{tabular}

q Follow-up mRS score - Admission mRS score $\leq 0$. 
\# Follow-up mRS score - Admission mRS score $>0$. 